Doi: $10.32481 /$ djph.2021.09.022

\title{
COVID-19 Vaccine Hesitancy in Delaware's Underserved Communities
}

\author{
Sharron Xuanren Wang, PhD: Department of Sociology and Criminal Justice, Delaware State \\ University \\ Nicole Bell-Rogers, EdD, FNP-C, RN: Department of Nursing, Delaware State University \\ Dorothy Dillard, PhD: Center for Neighborhood Revitalization and Research, Delaware State \\ University \\ Melissa A. Harrington, PhD: Delaware Institute of Science \& Technology, Delaware State \\ University
}

\begin{abstract}
Background and Objective: Vaccine hesitancy may be one of the greatest challenges to conquering the COVID-19 pandemic. Underserved communities across the U.S. have been suffering from the pandemic in unique ways, and vaccine hesitancy may exacerbate or prolong these issues. However, the prevalence of vaccine uptake and hesitancy in these vulnerable populations is unknown. The present study aimed to investigate: (1) prevalence of COVID vaccine uptake and COVID vaccine hesitancy in Delaware's underserved communities; (2) factors (i.e., demographic, socioeconomic characteristics, as well as COVID-related behaviors) associated with vaccine hesitancy; and (3) specific concerns about COVID vaccines. Materials and Methods: Data were extracted from a survey conducted in Delaware's underserved communities from March 4, 2021 to May 25, 2021. Logistic regression analyses were used to assess factors associated with vaccine hesitancy. Results: Results from our survey indicated that vaccine uptake is lower in Delaware's underserved communities than Delaware overall and the national average. In addition, a considerable proportion of participants were categorized as vaccine hesitant. We also found that being black increased the likelihood of vaccine hesitancy for the COVID-19 vaccine, which is consistent with prior studies on vaccine hesitancy. Results also indicated that having been tested for COVID in the past decreased the odds of vaccine hesitancy. However, we did not find that demographic or socioeconomic characteristics played a role in vaccine hesitancy in Delaware's underserved communities. Conclusion and Relevance: Our study represents a critial first step in understanding the determinants driving COVID vaccine uptake and hesitancy. Identifying key factors and causes for vaccine hesitancy may help in establishing novel strategies that counteract low vaccination rates in underserved communities.
\end{abstract}

\section{Introduction}

The COVID-19 pandemic has had a strong negative impact on the health and wellbeing of Americans since March 2020. Vaccines are a key element for decreasing the number of new infections, minimizing the likelihood of severe infections, and slowing down the pandemic. On Monday, May 25, 2021, President Biden announced that $50 \%$ of U.S. adults had been fully vaccinated against COVID-19. On the same day, about $42 \%$ of adults in Delaware had been fully vaccinated, and 53\% had received at least one dose. Delaware started to administer vaccines on a rolling basis starting December 15, 2020. The first eligible groups included healthcare personnel, emergency medical services agencies, and long-term care staff and residents (Phase 1A).

Starting January 19, 2021, eligibility expanded to 65 and older frontline essential workers (Phase 1B). The COVID vaccine became available to all Delawareans age 16 and older on April 6, 2021. ${ }^{1}$ By May 25, 2021, Delaware had administered 889,500 doses of COVID vaccines. Data from My Healthy Community, a Delaware Environmental Public Health Tracking Network, show that in Delaware more females than males have received at least one dose of the vaccine, and compared to Whites, Blacks have received at least one dose of the vaccine at a lower rate (i.e., $55 \%$ of Whites and $40 \%$ of Blacks by May 25, 2021). 
Racial and ethnic minorities have been not only disproportionately affected by the pandemic in terms of economic impact and mortality but also in terms of rate and speed of vaccination. A Kaiser Health News report found that Black Americans were vaccinated at rates 2 to 3 times below white Americans. ${ }^{2}$ The lower rate of vaccination among African Americans might be attributed to structural reasons (i.e., lower access to primary care, limited availability of vaccines in minority neighborhoods). Vaccine hesitancy may be one of the greatest challenges to conquering the COVID-19 pandemic. Vaccine hesitancy refers to the delay in acceptance or refusal of vaccines despite the availability of vaccine services. ${ }^{3}$ In 2019 , the World Health Organization (WHO) listed vaccine hesitancy as one of the top ten threats to global health. ${ }^{4}$ Vaccine hesitancy can be influenced by various factors, including race, ethnicity, age, sex, and socioeconomic background. ${ }^{5-8}$ Considering the history of medical abuse and racial discrimination in the healthcare setting toward Black Americans, many studies have suggested that Black Americans are more likely to express hesitancy toward COVID-19 vaccines. ${ }^{7}$ One study conducted in May 2020 using panel data estimated that $31 \%$ of the U.S. population expressed vaccine hesitancy. Recent studies have also reported a higher level of vaccine hesitancy among females and Black respondents. ${ }^{9}$ In addition, socioeconomic status was also negatively associated with vaccine acceptance. Another report using data from December 2020 suggested that $49 \%$ of Black Americans expressed COVID vaccine hesitancy, compared to 34\% of Whites. ${ }^{10}$

Ending the COVID-19 pandemic through vaccination programs may require a deeper understanding of who is hesitant and why. An array of factors contribute to vaccine hesitancy including mistrust of healthcare workers and the " $3 \mathrm{Cs}$," which include complacency, convenience, and confidence. ${ }^{11}$ Underserved communities across the U.S. have been suffering from the pandemic in many different ways. However, research and action plans geared toward helping these vulnerable populations are limited. ${ }^{12}$ Using data collected from March 2021 to May 2021, the current study explores vaccine hesitancy in Delaware's underserved communities. Specifically, this present study investigates: (1) the prevalence of COVID vaccine uptake and COVID vaccine hesitancy in Delaware's underserved communities; (2) factors (i.e., demographic, socioeconomic characteristics, as well as COVID-related behaviors) associated with vaccine hesitancy; and (3) concerns about COVID vaccines. The study hopes to shed light on the determinants of vaccine hesitancy, thus providing new strategies for outreach among disadvantaged communities to promote COVID vaccination and narrow health disparities caused by the pandemic.

\section{Recruitment}

The study was approved by the Institutional Review Board at Delaware State University. All study participants were 18 years of age or older and were residents of Delaware's most underserved communities as defined by health and socio-demographic indicators. We defined underserved communities as areas, geographically indicated by census tract, experiencing high rates of poverty, unemployment, and health disparities. Delaware's Division of Public Health (DE DPH) adopted the Community Health Index (CHI) as the common indicator for characterizing community health at the census tract level. The $\mathrm{CHI}$ is a composite score derived from life expectancy, infant mortality rate, percentage of high school graduates, and child poverty rates. It serves as a proxy of community health. DE DPH used the CHI scores to identify the Delaware communities with the greatest need in addressing the social determinants of health that drive health disparities. As expected, these communities have concentrated poverty, limited 
health resources, and large minority populations. We have selected these communities as our study sites because they experience the greatest need for intervention specific to COVID-19 and health disparities in general.

We collaborated with two trusted community health advocacy agencies, Wilmington Hope Commission (WHC) and the Sussex County Health Coalition (SCHC). WHC and SCHC currently serve as key coordinating organizations in Delaware's COVID-19 response. They also have established partner networks comprised of community-based health providers and clinics, non-profit organizations, and faith-based organizations based in our communities of interest. The community-health providers and clinics have established trust and a good rapport among the residents. We worked with WHP and SCHS to develop a recruitment protocol, recruitment materials, and information appropriate for the residents of the study sites; to establish partnerships with the community-based organizations and clinics serving the residents; and to maintain working relationships with the study sites.

\section{Data and Methods}

Data were extracted from a survey conducted from March 4, 2021, to May 25, 2021. The survey contained questions about demographics, socioeconomic characteristics, COVID-related beliefs and practices, general health, and testing and vaccine-related questions. Questions on the survey were adopted from the COVID-19 Community Response Survey developed by John Hopkins University and the NIH Common Data Elements (CDEs). Surveys were conducted electronically through REDcap on iPads at the study sites. Nurses, student interns, staff, and participants wore masks at all times, sanitized their hands and IPADs frequently, and remained 6-feet apart. By May 25, 2021, 307 participants have been recruited in the study. Figure 1 displays a map showing the residency of participants based on their zip code. After excluding participants residing outside of Delaware, age younger than 18 , and Hispanics from the study (due to the very small sample size of Hispanics, we did not examine this sample characteristic in order to protect the identities of the study participants), 293 participants remained in the analysis. Figure 1 was created based on the zip codes provided by 266 participants. Forty-two participants did not indicate zip code, and their location was based on zip codes of the study sites.

Figure 1. Map of Participants' Residence in Delaware Based on Zip Code 
Doi: $10.32481 /$ djph.2021.09.022

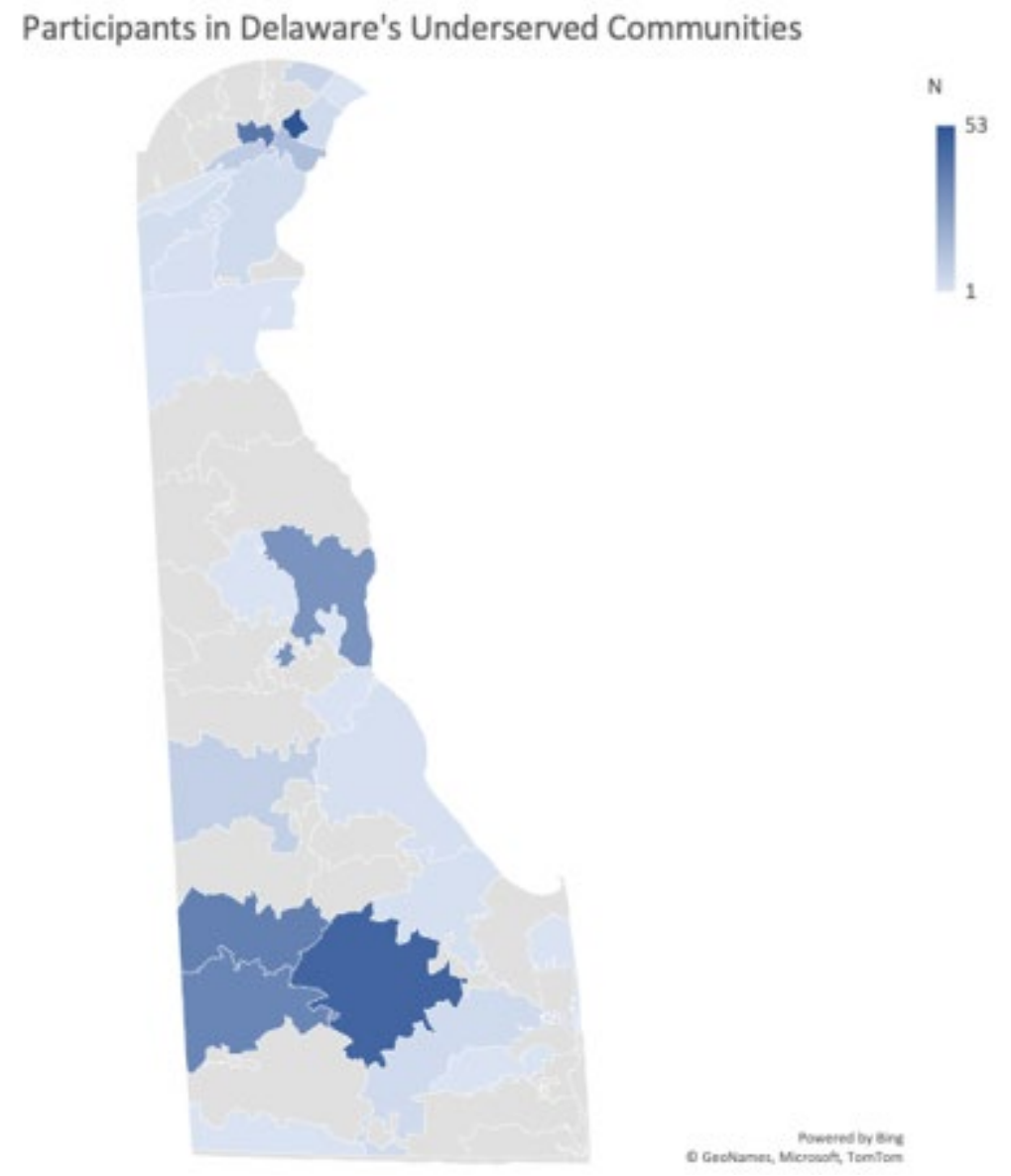

\section{Measures}

The outcome variable of interest is a binary variable vaccine hesitancy (yes $=1 / \mathrm{no}=0$ ). Vaccine hesitancy was operationalized with a multiple-choice question, "Why have you not received your COVID vaccine?" The answers to the question included:

- I am not qualified;

- I do not want to get vaccinated;

- I am qualified, but I had a hard time finding available vaccines;

- I am unaware of the availability of the vaccine;

- Other;

- Prefer not to answer; and

- Don't know.

Respondents who chose the answer "I do not want to get vaccinated," "Prefer not to answer," and "Don't know" were coded as vaccine hesitant, consistent with prior research. ${ }^{9}$ We further assessed the open-ended specified answers for those who picked "Other." We coded the answers expressing any vaccine hesitancy to a numerical value of one (1). 
A set of socio-demographic characteristics are used as covariates. We included information on race (Black and others), age, educational level, current employment status, whether participants experienced job loss during the pandemic, whether participants had health insurance, family income in 2020, and the number of people living in the household. We used Black (Yes = 1; No $=0)$ as an indicator for race. Due to the small sample size, we grouped respondents identified as White, Asian, Native American, Pacific Islander, and other race into "others." It should be noted that the majority of respondents coded as "others" were whites (about 80\%). We also included whether respondents have been tested for COVID infection (yes $=1$; no $=0$ ) and whether respondents have ever tested positive for COVID infection (yes $=1$; no $=0$ ) as covariates.

Lastly, we included COVID-related behaviors and practices. The questions were extracted from the COVID-19 Community Response Survey. The questions asked whether the respondents have made any changes to their lifestyles or daily activities because of the COVID-19 pandemic. The list includes:

- More handwashing than usual (yes=1/no=0);

- More use of hand sanitizer than usual (yes= $1 / \mathrm{no}=0)$;

- More cleaning in your home than usual (yes= $1 / \mathrm{no}=0)$;

- More disinfecting surfaces in household than usual (yes $=1 / \mathrm{no}=0)$;

- Disinfecting or wiping down groceries $(\mathrm{yes}=1 / \mathrm{no}=0)$;

- Disinfecting or wiping down mail or packages (yes $\left.=1 / \mathrm{no}^{=}=0\right)$;

- Stocking up on food and supplies; avoiding or cancelling domestic traveling (yes= $1 / \mathrm{no}=0)$;

- Not ordering take out from restaurants $(\mathrm{yes}=1 / \mathrm{no}=0)$; and

- Wearing a mask when out in public $(\mathrm{yes}=1 / \mathrm{no}=0)$.

We further generated a variable named the COVID Cautiousness Scale to indicate the level of cautious behaviors during the pandemic. Specifically, we added up all the COVID-related behavioral questions for which the respondent indicated a yes. Each yes was assigned a value of one (1). Therefore, score on the COVID Cautiousness Scale range from 0 to 11. A higher score indicates the respondent displayed more cautious behavior during the pandemic. It should be noted that this scale has not been validated as a reliable measure of COVID cautiousness for the general population. The investigators simply used this score as a proxy to measure the level of cautiousness for each participant.

\section{Methods}

To investigate prevalence of COVID vaccine uptake and COVID vaccine hesitancy in Delaware's underserved communities, we calculated the percentages of respondents who received COVID vaccines and those who reported that they would not get the vaccines. We used the logistic regression model to predict the factors associated with vaccine hesitancy. Lastly, we ran chi-square analyses to explore whether vaccine-related concerns in the underserved communities differed by race. It should be noted that missing data is a common issue in research focused on hard-to-reach populations. For some variables, we had a relatively large number of missing responses. Some of the questions might be too sensitive to answer for our participants 
(i.e., income) or the available answers were not applicable (i.e., number of people in household for homeless individuals). Under these circumstances, the missing variables might not be missing completely at random (MCAR). Therefore, a complete case analysis might yield biased results. In this preliminary study, we used a missing indicator approach to handle missing responses in the analysis. The missing indicator approach is suggested to be efficient when the sample size is relatively small and thus may be reasonably applied to the present study. ${ }^{13}$

\section{Results}

Table 1 displays characteristics of the sample. The percentage of missing responses for the covariates was included. The average age of the sample was $44.36(\mathrm{SD}=14.55)$. Blacks were oversampled; $73 \%$ of the sample identified as black, and $27 \%$ identified as white, Asian, Native American, and others. We grouped all the non-black respondents into "other races." About half of the respondents identified as male and half as female.

Table 1. Study Characteristics

\begin{tabular}{lc}
\hline Sample size & 293 \\
Socioeconomic and demographic characteristics & \\
Race (\%) & \\
Black & 73.13 \\
Others (Whites, Asian, Native American, and Others) & 26.87 \\
Total & 100 \\
Mean age & $44.36(\mathrm{SD}=14.55)$ \\
Missing on age (\%) & 24.49 \\
Sex & \\
Male & 48.98 \\
Female & 48.98 \\
Missing & 2.04 \\
Total & 100 \\
Education (\%) & \\
Less than high school & 21.09 \\
High school diploma & 42.86 \\
Some college degree and higher & 31.63 \\
Missing & 4.42 \\
Total & 100 \\
Current employment status & \\
Working & 40.48 \\
Unemployed & 24.49 \\
Not in labor force & 24.83 \\
Missing & 10.20 \\
Total & 100 \\
Health Insurance (\%) & 81.98 \\
Job loss during COVID (\%) & 33.67 \\
$\quad$
\end{tabular}


Doi: 10.32481/djph.2021.09.022

Family income in $2020<\$ 20,000(\%)$

47.96

\# of people in the household

Missing on \# of people in household (\%)

$2.44(\mathrm{SD}=1.73)$

27.21

COVID Cautiousness scale

$8.56(\mathrm{SD}=3.31)$

COVID testing questions

Tested for COVID (\%)

Tested Positive for COVID among tested (\%)

\section{COVID Vaccine Questions}

Covid Vaccine (\%)

30.38

Vaccine hesitancy among not vaccinated (\%)

60.29

Vaccine hesitancy among all sample (\%)

Regarding the highest educational level, $21 \%$ reported less than high school, $43 \%$ received a high school diploma, and $32 \%$ reported having at least some college degree. In terms of current employment status, $41 \%$ reported that they were working, $25 \%$ were unemployed, and $25 \%$ were not in the labor force. Moreover, $48 \%$ of our sample reported that their 2020 household family income was less than $\$ 20,000$. In terms of job status during COVID, $34 \%$ of the respondents reported that they experienced job loss. The average number of people living in the household was $2.44(\mathrm{SD}=1.73)$, and $82 \%$ of respondents had health insurance. To measure participants' behaviors and cautiousness during the pandemic, we constructed a COVID cautiousness scale using a set of questions on the survey. The average score was $8.56(\mathrm{SD}=3.31)$.

Regarding COVID testing and vaccine status, $65 \%$ of our sample have tested for COVID infection in the past, and $15 \%$ of the sample indicated that they have at some point received a positive COVID test result. Thirty percent of our sample has received at least one dose of the COVID vaccine. It should be noted that this number is smaller than the average reported for Delaware overall, $53 \%$. In terms of vaccine hesitancy, $60 \%$ of the unvaccinated sample were categorized as vaccine hesitant ( $42 \%$ of the total sample).

Table 2 presents odds ratios of the logistic regression model predicting vaccine hesitancy. The outcome of interest was the binary variable indicating vaccine hesitancy. Vaccine hesitancy was coded 1 and otherwise 0 . The covariates included demographics (i.e., race, age, square of age, and sex), socioeconomic characteristics (i.e., educational level, current employment status, health insurance, job loss experience during the pandemic, and number of people in the household), COVID-related behavior and testing characteristics (i.e., COVID Cautiousness scale, tested for COVID, and tested positive for COVID). We also included indicators for survey month to control for the changing vaccine policies over time. Finally, a set of variables indicating missing data were included as covariates.

Table 2. Logistic Regression Predicting Vaccine Hesitancy

\begin{tabular}{lccc}
\hline & Odds Ratio & Robust SE & P value \\
\hline Black & $2.0161^{*}$ & 0.6689 & 0.035 \\
Others & REF & REF & REF \\
Age & 1.0994 & 0.1066 & 0.328 \\
Square of age & 0.9982 & 0.0012 & 0.129 \\
Missing on age & $0.5038+$ & 0.1924 & 0.073 \\
\hline
\end{tabular}


Doi: 10.32481/djph.2021.09.022

\begin{tabular}{lccc}
\hline Male & 1.4078 & 0.407 & 0.237 \\
Missing on sex & 0.9255 & 0.9394 & 0.939 \\
Less than high school & REF & REF & REF \\
High school diploma & 0.8860 & 0.3461 & 0.757 \\
Some college degree and higher & 0.7654 & 0.3329 & 0.539 \\
Missing on education & $0.1019^{*}$ & 0.1007 & 0.021 \\
Currently working & 1.2171 & 0.4741 & 0.614 \\
Currently unemployed & 1.4204 & 0.5705 & 0.382 \\
Currently not in labor force & REF & REF & REF \\
Missing on employment information & 1.6553 & 0.8945 & 0.351 \\
Have health insurance & 1.0315 & 0.4275 & 0.940 \\
Job loss during COVID & 1.1365 & 0.352 & 0.680 \\
Family income in 2020 $<$ 20,000 & 1.6635 & 0.6004 & 0.159 \\
\# of people in the household & $0.8482+$ & 0.0835 & 0.095 \\
Missing on \# of people in household & $0.4608+$ & 0.2019 & 0.077 \\
COVID Cautiousness scale & 1.0142 & 0.0469 & 0.760 \\
Tested for COVID & $0.4340^{*}$ & 0.1499 & 0.016 \\
Tested Positive for COVID among tested & 0.8794 & 0.3723 & 0.762 \\
Survey was conducted in & & & \\
$\quad$ Mar-2021 & REF & REF & REF \\
Apr-2021 & 1.7026 & 0.6262 & 0.148 \\
May-2021 & 1.2958 & 0.5253 & 0.523 \\
Constant & 0.3202 & 0.6528 & 0.576 \\
\hline Pse R2 - 0.1909
\end{tabular}

Pseudo R2 $=0.1909$

$\mathrm{N}=293$

Results from the logistic regression estimates suggested that being black $(\mathrm{OR}=2.02, \mathrm{p}=0.035)$ increased the odds of vaccine hesitancy by $100 \%$ compared to other races while holding all the covariates constant. Another noteworthy significant result involved COVID testing ( $\mathrm{OR}=0.4340$, $\mathrm{p}=0.016$ ). Results indicated that if the respondents had tested for COVID in the past, the odds of vaccine hesitancy decreased by $57 \%$ while holding all the covariates constant. The missing indicator on education is also statistically significant at the $p<0.05$ level, further indicating that missingness on education might have significant effect on vaccine hesitancy. Other than being black and having tested for COVID, we did not find other significant effects of demographic and socioeconomic characteristics on vaccine hesitancy.

We took the mean of each of the covariates in the logistic regression model and estimated the predicted probabilities of vaccine hesitancy for blacks and others (see Figure 2). The predicted probability of vaccine hesitancy for blacks was $0.42(\mathrm{SE}=0.04)$ and for others was 0.26 $(\mathrm{SE}=0.06)$ for a typical Delawarean living in underserved communities.

Figure 2. Predicted Probabilities of Vaccine Hesitancy by Race in DE's Underserved Communities 


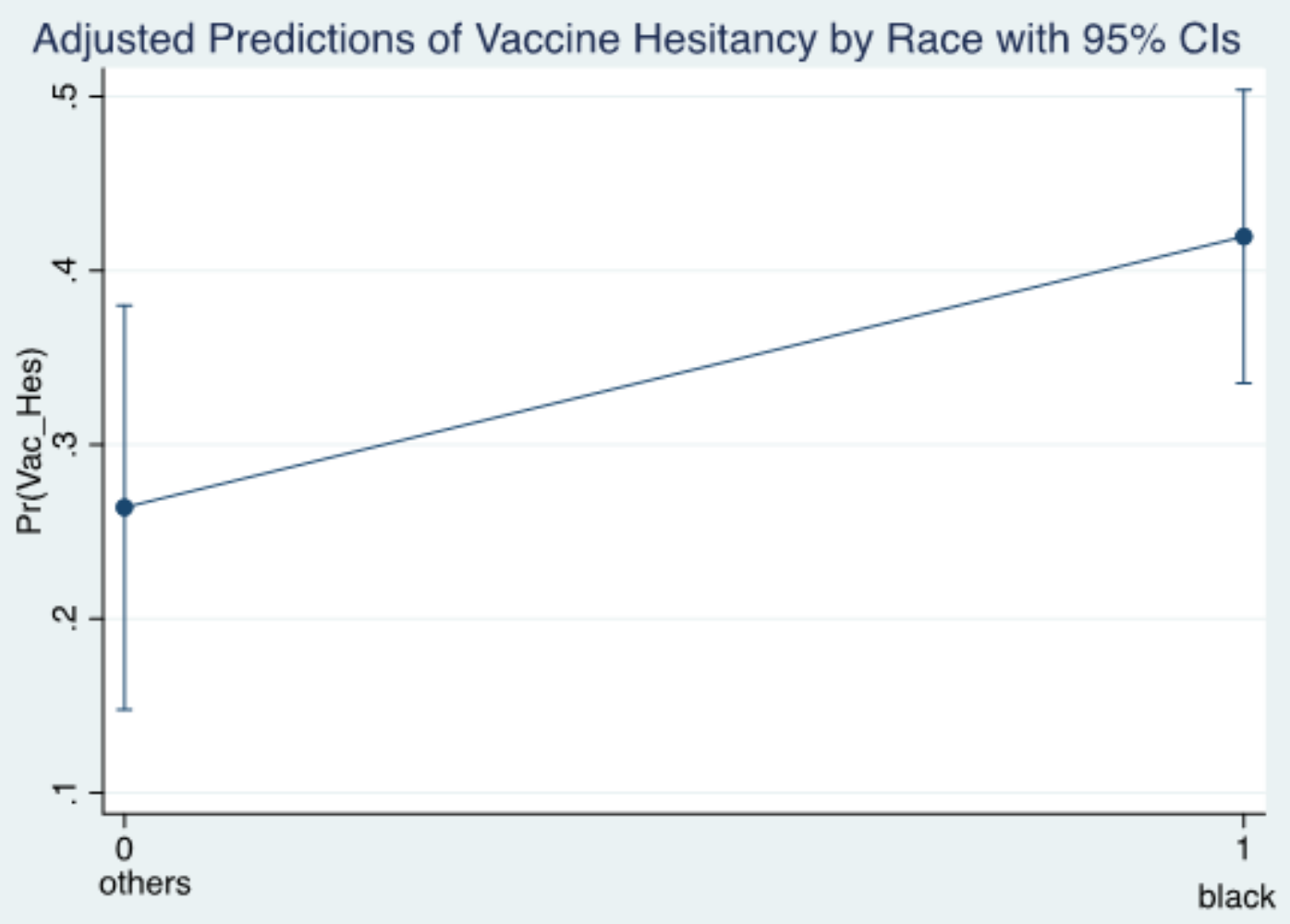

Finally, our survey included a set of questions about concerns potentially contributing to vaccine hesitancy among blacks in DE's underserved communities. The set of concerns included:

(1) I am allergic to vaccines;

(2) I don't like needles;

(3) I'm not concerned about getting sick from COVID;

(4) I'm concerned about side effects;

(5) I don't think vaccines work well;

(6) I don't trust that the vaccine will be safe;

(7) I don't believe the COVID pandemic is as bad as they reported;

(8) I don't want to pay for it; and

(9) I don't know enough about how well the vaccine works.

A simple percentage calculation reported that among those respondents who expressed vaccine hesitancy, 33\% were concerned about side effects, 25\% did not trust the vaccine would be safe, and $21 \%$ indicated that they did not like needles.

We conducted chi-square analyses to evaluate whether there were any significant differences in vaccine-related concerns between blacks and non-blacks (see Table 3). At the $p<0.1$ level, the chi-square test result suggested that more blacks expressed concern that the vaccine would not work well compared to others in the sample $(\mathrm{Chi} 2=3.24, \mathrm{p}=0.072)$. Another significant result was that more non-black respondents indicated that they did not believe the pandemic was as bad as reported compared to blacks. 
Doi: $10.32481 /$ djph.2021.09.022

Table 3. Concerns of COVID Vaccine Among Respondents Who Expressed Vaccine Hesitancy

\begin{tabular}{lccccc}
\hline & $\begin{array}{c}\text { Sample } \\
(\%)\end{array}$ & $\begin{array}{c}\text { Black } \\
(\%)\end{array}$ & $\begin{array}{c}\text { Others } \\
(\%)\end{array}$ & $\mathrm{Chi}^{2}$ test & P value \\
\hline (1) I'm allergic to vaccines & 5.69 & 7.22 & 0 & 1.9895 & 0.158 \\
(2) I don't like needles & 21.14 & 18.56 & 30.77 & 1.8345 & 0.176 \\
(3) I'm not concerned about getting & & & & & \\
sick from COVID & 13.01 & 11.34 & 19.23 & 1.1281 & 0.288 \\
(4) I'm concerned about side effects & 32.52 & 31.96 & 34.62 & 0.0659 & 0.797 \\
$\begin{array}{l}\text { (5) I don't think vaccines work well } \\
\text { (6) I don't trust that the vaccine will }\end{array}$ & 8.94 & 11.34 & 0 & $\mathbf{3 . 2 3 8 0}+$ & 0.072 \\
be safe & 25.2 & 23.71 & 30.77 & 0.5418 & 0.462 \\
(7) I don't believe the covid pandemic & & & & & \\
is as bad & 2.44 & 1.03 & 7.69 & $\mathbf{3 . 8 2 3 6 +}$ & 0.051 \\
(8) I don't want to pay for it & 3.25 & 3.09 & 3.85 & 0.037 & 0.847 \\
(9) I don't know enough about how & & & & & \\
well the vaccine works & 18.7 & 16.49 & 26.92 & 1.4667 & 0.226 \\
$\mathrm{~N}$ & 123 & 97 & 26 & & \\
\hline
\end{tabular}

\section{Discussion}

The COVID-19 pandemic has caused a major disruption to people's lives on a global scale. COVID testing and vaccines are essential to combat the pandemic. Recent research has suggested that economically vulnerable communities across the country have been more severely affected by the pandemic. Testing and vaccine rates among these communities remain lower than other communities. It is important to explore the factors that potentially contribute to vaccine hesitancy in vulnerable communities. Our study represents a first step to understanding the determinants driving COVID vaccine uptake and hesitancy. Identifying key factors and causes for vaccine hesitancy and ways to counteract low vaccination uptake are primary steps to addressing this issue. Interventions to address and improve uptake levels specific to this study may inform other communities in their pursuits to increase vaccine uptake while also providing incite on how to reduce health disparities among vulnerable populations now and in the future.

Results from our survey indicated that vaccine uptake is lower in Delaware's underserved communities than Delaware overall and the national average. In addition, a considerable proportion of participants were categorized as vaccine hesitant. We also found that being Black increased the likelihood of vaccine hesitancy for the COVID-19 vaccine, which is consistent with prior studies on vaccine hesitancy. However, we did not find that demographic or socioeconomic characteristics play a role in vaccine hesitancy in Delaware's underserved communities.

Underserved communities show lower rates of COVID vaccination when compared to others, and many of the respondents in our survey indicated that they would not get the vaccine. These attitudes, along with other risk factors, could prolong the health crisis for underserved communities. Our study has important implications for public health. For instance, it may be beneficial for government and public health services to reach out to vulnerable communities and spread awareness about the safety, effectiveness, and importance of getting vaccinated for COVID-19. Harrison indicated that vaccine hesitancy surrounding the COVID-19 vaccine 
involves several subthemes, including public and individual concerns about vaccine safety and effectiveness, lack of trust regarding vaccination efforts, vaccine misinformation, and the push for vaccine uptake. ${ }^{14}$ Many of our participants indicated that they do not think the vaccine works well or do not know enough about how well it works. Such knowledge might be limited among vulnerable communities for a variety of reasons, such as lower health literacy; lack of access to primary care; socioeconomic status; political, religious beliefs and values; and lack of outreach among these communities. ${ }^{15}$

\section{Public Health Implications}

Specific efforts to understand the surrounding community and causative factors for hesitancy will be further explored as our survey continues. Potential solutions to vaccine hesitancy might involve increased communication on the benefits and safety of vaccines, in addition to targeted approaches that address specific hesitancy issues in a given community. Other strategies that can be employed to reduce hesitancy include encouraging conversation among local community members, neighbors, co-workers, friends, and family. During these conversations, those who have received the vaccine can share personal accounts of their experience. This particular strategy has been shown to be effective at reducing hesitancy at the local level. ${ }^{14}$

Notably, strategies to combat hesitancy would greatly benefit from collaborations with trusted community partners (academic, healthcare providers, and community agencies) and the implementation of public health initiatives. ${ }^{16}$ Such efforts would help build targeted public health approaches between underserved minority communities and the medical community to address the longstanding health disparities that predate the COVID-19 pandemic. While COVID-19 is a public health emergency of international concern, as declared by the World Health Organization (WHO) in July 2020, efforts to combat the pandemic and its sequelae must begin at the local level. ${ }^{14,17}$ The present study demonstrates the benefits of working with community partners in determining vaccine hesitancy issues, and it is believed that the same community partnerships may aid in carrying out strategies to alleviate vaccine concerns. There is a great need for innovative public health projects/programs that circulate timely, evidence-based information to targeted audiences (e.g., social media, online platforms, local news outlets, occupational health clinics, and other traditional methods) while also focusing on community engagement. Key elements to reduce vaccine hesitancy should be practical and aimed toward minority ethnic groups with low vaccine uptake to reduce misinformation and increase confidence. ${ }^{16}$

\section{Disclosures}

This project is supported by the National Institute of Health (Grant number: 3 P20 GM10365309S1)

\section{Acknowledgements}

The authors would like to thank Mr. Matthew Billie, student interns, and student nurses for their support in data collection. They would also like to thank the community partners Wilmington Hope Commission and the Sussex County Health Coalition for their support. Lastly, the authors would like to thank the Delaware CTR ACCEL for providing REDcap access to conduct the survey and Drs. Suzanne McCahan and Stephan DiDonato for their assistance using REDcap. 
Doi: $10.32481 /$ djph.2021.09.022

\section{References}

1. Carney, J. (2021, Mar 30). COVID-19 Vaccination Program Will Open to Delawareans 16+ on April 6. Retrieved from: https://news.delaware.gov/2021/03/30/covid-19-vaccinationprogram-will-open-to-delawareans-16-on-april-6/

2. Recht, H., \& Weber, L. (2021, Jan). Black Americans are getting vaccinated at a lower rates than White Americans. Kaiser Health News. Retrieved from:

https://khn.org/news/article/black-americans-are-getting-vaccinated-at-lower-rates-thanwhite-americans/

3. Butler, R., \& MacDonald, N. E., \& the SAGE Working Group on Vaccine Hesitancy. (2015, August 14). Diagnosing the determinants of vaccine hesitancy in specific subgroups: The Guide to Tailoring Immunization Programmes (TIP). Vaccine, 33(34), 4176-4179. PubMed https://doi.org/10.1016/j.vaccine.2015.04.038

4. World Health Organization. (2019). Ten Threats to Global Health 2019. Retrieved from: https://www.who.int/news-room/spotlight/ten-threats-to-global-health-in-2019

5. Fisher, K. A., Bloomstone, S. J., Walder, J., Crawford, S., Fouayzi, H., \& Mazor, K. M. (2020, December 15). Attitudes toward a potential SARS -CoV-2 vaccine: A survey of US adults. Annals of Internal Medicine, 173(12), 964-973. PubMed https://doi.org/10.7326/M20-3569

6. Kreps, S., Prasad, S., Brownstein, J. S., Hswen, Y., Garibaldi, B. T., Zhang, B., \& Kriner, D. L. (2020, October 1). Factors associated with us adults' likelihood of accepting COVID-19 Vaccination. JAMA Network Open, 3(10), e2025594. PubMed https://doi.org/10.1001/jamanetworkopen.2020.25594

7. Pogue, K., Jensen, J. L., Stancil, C. K., Ferguson, D. G., Hughes, S. J., Mello, E. J., . . . Poole, B. D. (2020, October 3). Influences on attitudes regarding potential COVID-19 vaccination in the United States. Vaccines, 8(4), 582. PubMed https://doi.org/10.3390/vaccines 8040582

8. Troiano, G., \& Nardi, A. (2021, May). Vaccine hesitancy in the era of COVID-19. Public Health, 194, 245-251. PubMed https://doi.org/10.1016/j.puhe.2021.02.025

9. Doherty, I., Pilkington, W., Brown, L., Billings, V., Hoffler, U., Paulin, L., \& Kumar, D. (2021). COVID-19 vaccine hesitancy in underserved communities of North Carolina. MedRxiv. DOI:

10. Karpman, M., Kenney, G. M., Zuckerman, S., Gonzalez, D., \& Courtot, B. (2021). Confronting COVID-19 vaccine hesitancy among nonelderly adults. Robert Wood Johnson Foundation and Urban institute Retrieved from: https://www.rwjf.org/en/library/research/2021/02/confronting-covid-19-vaccine-hesitancyamong-nonelderly-adults. html

11. MacDonald, N. E., \& the SAGE Working Group on Vaccine Hesitancy. (2015, August 14). Vaccine hesitancy: Definition, scope and determinants. Vaccine, 33(34), 4161-4164. PubMed https://doi.org/10.1016/j.vaccine.2015.04.036

12. Kantamneni, N. (2020). The impact of the COVID-19 pandemic on marginalized populations in the United States: A research agenda. 
13. Sperrin, M., Martin, G. P., Sisk, R., \& Peek, N. (2020, September). Missing data should be handled differently for prediction than for description or causal explanation. Journal of Clinical Epidemiology, 125, 183-187. PubMed https://doi.org/10.1016/j.jclinepi.2020.03.028

14. Harrison, J., Berry, S., Mor, V., \& Gifford, D. (2021, June). "Somebody like me:” Understanding COVID-19 vaccine hesitancy among staff in skilled nursing facilities. Journal of the American Medical Directors Association, 22(6), 1133-1137. PubMed https://doi.org/10.1016/j.jamda.2021.03.012

15. Lackner, C. L., \& Wang, C. H. (2021, August). Demographic, psychological, and experiential correlates of SARS-CoV-2 vaccination intentions in a sample of Canadian families. Vaccine: $X, 8,100091-100091$. PubMed https://doi.org/10.1016/j.jvacx.2021.100091

16. Robertson, E., Reeve, K. S., Niedzwiedz, C. L., Moore, J., Blake, M., Green, M., . . . Benzeval, M. J. (2021, May). Predictors of COVID-19 vaccine hesitancy in the UK household longitudinal study. Brain, Behavior, and Immunity, 94, 41-50. PubMed https://doi.org/10.1016/j.bbi.2021.03.008

17. World Health Organization. (2020, December 24). Ten global health issues to track in 2021. Retrieved from: https://www.who.int/news-room/spotlight/10-global-health-issues-to-trackin-2021

Copyright (c) 2021 Delaware Academy of Medicine / Delaware Public Health Association.

This is an Open Access article distributed under the terms of the Creative Commons Attribution Non-Commercial License (https://creativecommons.org/licenses/by-nc-nd/4.0/) which permits unrestricted non-commercial use, distribution, and reproduction in any medium, provided the original work is properly cited. 